\title{
Low-Loss Imaging of Defect Structures in Two Dimensional Materials Using Aberration Corrected Scanning Transmission Electron Microscopy.
}

\author{
Mark P. Oxley ${ }^{1,2}$, Myron D. Kapetanakis ${ }^{2,1}$, Wu Zhou ${ }^{1}$, Juan-Carlos Idrobo ${ }^{3}$, Sokrates T. Pantelides ${ }^{2,1}$ \\ 1. Material Science and Technology Division, Oak Ridge National Laboratory, Oak Ridge, TN, USA. \\ 2. Department of Physics and Astronomy, Vanderbilt University, Nashville, TN, USA. \\ 3. Center for Nanophase Materials Sciences Division, Oak Ridge National Laboratory, Oak Ridge, TN, \\ USA.
}

Aberration correction of electron optics has provided not only higher resolution, but also higher contrast. This is particularly true for scanning transmission electron microscopes (STEMs) operating at lower accelerating voltages, which has allowed atomic resolution of 2-dimensional materials using both annular dark field (ADF) and electron energy loss spectroscopy (EELS) based on core-loss excitations $[1,2]$. It has until recently been assumed that atomic resolution images based on low-loss or valance EELS (VEELS) would not be possible, due to the assumed delocalization of the inelastic interaction involved. This has recently been shown not to be the case, with atomic resolution VEELS maps obtained from graphene using aberration corrected STEM and explained using first principles theory [3]. In this presentation we discuss the influence of defects and impurities in 2-dimensional materials on experimental VEELS maps, and how the underlying contrast mechanisms can be explained using a combination of density functional theory (DFT) and dynamical electron scattering theory [4].

In figure 1 (a) we show an ADF image of graphene with a 4-fold coordinated silicon impurity, acquired using ORNL's Nion UltraSTEM operating at $60 \mathrm{kV}$. Shown in figure 1 (b) and (c) are VEELS maps formed by integrating the VEELS signal over energy ranges of 11-22 eV and 22-44 eV. Figure 1 (b) is dominated by the signal from the site of the Si atom, whereas for 1 (c) the intensity on this site is lower than the surrounding graphene. Such a reversal of contrast cannot be explained by recourse to phenomena such as preservation of elastic contrast. This can only be understood by the application of detailed theory describing both the image formation and the inelastic VEEL excitations.

For the experimental conditions used here, the image is well described by the convolution of the STEM probe with the local inelastic scattering potential. The scattering potential for a particular transition from the initial state $i$ to the excited state $f$ may be written as

$$
V_{i, f}(\mathbf{r}) \propto \frac{\left|\left\langle\psi_{f}(\mathbf{r})\left|e^{-2 \pi \mathbf{i q} \cdot \mathbf{r}}\right| \psi_{i}(\mathbf{r})\right\rangle\right|^{2}}{\mathbf{q}^{4}}
$$

where the wave functions $\psi$ describe the initial and final states of the transition and $\mathbf{q}$ is related to the momentum transfer of the scattered electron. The initial and final states are calculated using DFT within the projector augmented wave (PAW) approach as implemented in VASP. The resulting matrix elements are calculated in full rather than applying the more common dipole approximation. Images are then formed by convolving the resulting potential with the calculated STEM probe intensity, including corrections for temporal and spatial incoherence.

The results of these calculations are shown in figure 2. Both the bright feature at lower energy and the contrast reversal at higher energy losses are well reproduced. Further to this result, calculations have shown that the coordination of the Si impurity within the graphene lattice is important. While both 3- 
fold and 4-fold impurities reproduce the bright features at lower energies, only 4-fold coordination produces the correct contrast between $22-44 \mathrm{eV}$.

The dependence of VEELS contrast on local bonding offers new way of studying the properties of 2dimensional materials. With the development of the latest generation of monochomators, even finer details are potentially available for closer study using this approach on a range of 2-dimensional materials with many applications to the vital field of renewable energy.

\section{References}

[1] Krivanek et al., Nature, 462, 7228 (2010).

[2] Zhou et al., Microscopy and Microanalysis, 18, 1342 (2012).

[3] Kapetanakis et al., Phys. Rev. B, 92, 125147 (2015).

[4] Kapetanakis et al., under review.

[5] This research was supported by DOE Grant No. DE-FG02-09ER46554 (MDK, STP), by the U.S. Department of Energy Office of Science, Basic Energy Sciences, Materials Sciences and Engineering Division (MPO, WZ), the Center for Nanophase Materials Sciences (CNMS), which is sponsored at ORNL by the Scientific User Facilities Division, Office of Basic Energy Sciences, U.S. DOE (JCI). Numerical calculations were performed at the National Energy Research Scientific Computing Center (NERSC), which is supported by the Office of Science of the U.S. Department of Energy under Contract No. DE-AC02-05CH11231.
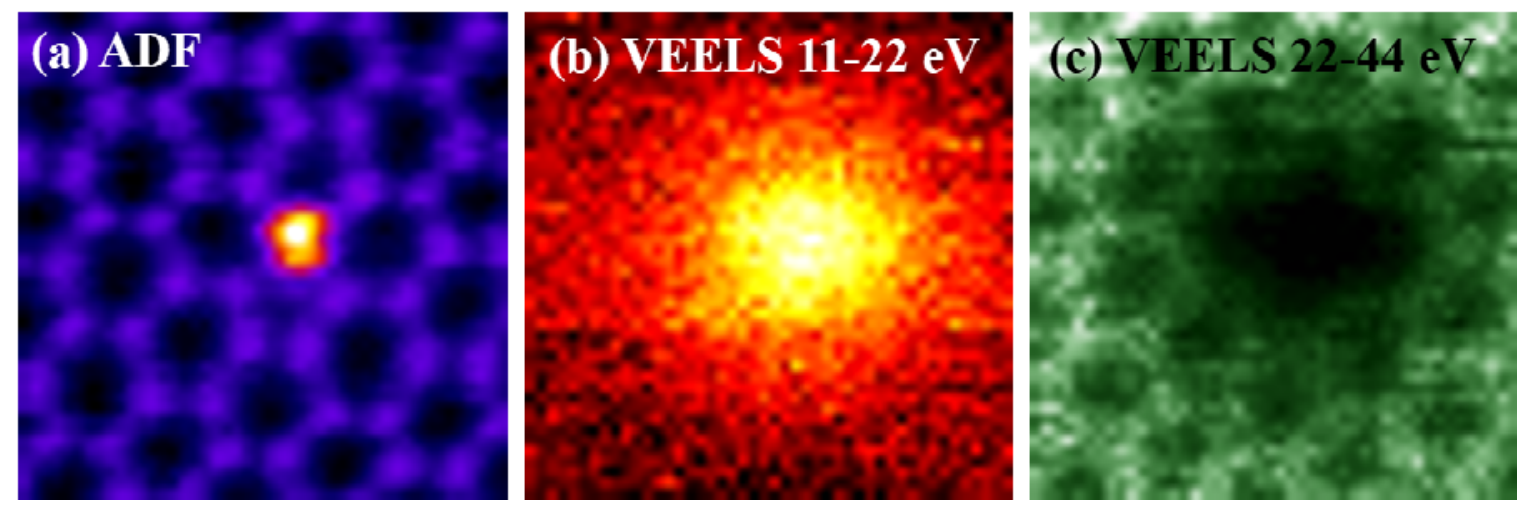

Figure 1. (a) ADF image of a Si impurity in graphene. Also shown are simultaneously obtained VEELS images covering the energy ranges of (b) $11-22 \mathrm{eV}$ and (c) $22-44 \mathrm{eV}$.
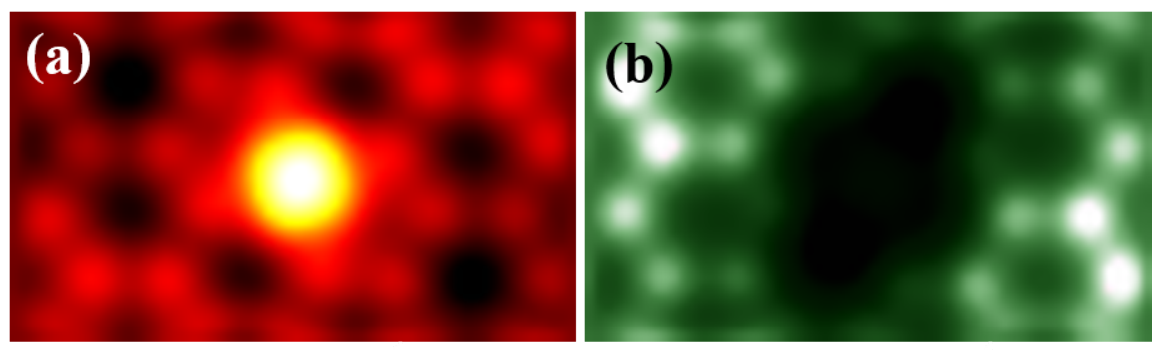

Figure 2. Simulated VEELS images of a Si impurity in graphene for energy losses of (a) 11-22 eV and (b) $22-44 \mathrm{eV}$. 